\title{
Surgical Outcome and Histopathological Findings of Feline Corneal Sequestrum
}

\author{
Sarbani Hazra ${ }^{1 *}$, Sabyasachi Konar ${ }^{1}$, Vinod Kumar ${ }^{1}$, Aditya Konar ${ }^{2}$ \\ ${ }^{1}$ Department of Surgery \& Radiology, West Bengal University of Animal \& Fishery Sciences, 37 \& 68, Khudiram Bose Sarani, Kolkata, West Bengal -700037, India \\ ${ }^{2}$ CSIR-Indian Institute of Chemical Biology, Kolkata, West Bengal 700032, India
}

Received: August 04, 2015; Accepted: December 20, 2015; Published: January 01, 2016

*Corresponding author: Sarbani Hazra, Department of Surgery \& Radiology, West Bengal University of Animal \& Fishery Sciences, 37 \& 68, Khudiram Bose Sarani, Kolkata-700037, India, E-mail: shazrakon@yahoo.co.in

\begin{abstract}
Purpose: To evaluate the surgical outcome and histological features of feline corneal sequestrum following superficial keratectomy.

Materials \& Methods: Corneal sequestration was diagnosed in the left eye of a 4-year-old neutered female cat. After obtaining appropriate history, slit lamp biomicroscopy, Schirmer's tear test and fluorescein dye test was performed. Superficial keratectomy was performed under general and topical anesthesia. Post-operative care and management for the healing of the wound bed was instituted. The respected tissue was processed for histopathological evaluation. A follow-up for up to 1 month was done to assess the surgical outcome.

Result: The slit lamp biomicroscopy showed the involvement of the sequestration up to mid-stromal layer. Tear wetting following Schirmer's tear test was normal, and the sequestrum was negative to fluorescein stain except at the edges of the lesion. Superficial keratectomy successfully performed to remove the sequestered tissue. The histopathological findings showed stromal fibrosis. One month follow-up showed good healing of wound bed associated with mild scarring. An initiation of a new nidus of sequestration away from the previous one was evidenced.
\end{abstract}

Conclusion: Feline corneal sequestration has been successfully treated surgically, an insight on the histological characteristics of sequestered tissue was obtained, and a recurrence of sequestration in a new site has been observed.

\section{Introduction}

Corneal sequestration in feline has varied aetiology i.e. hereditary [1], feline herpes Virus -1 [2,3] conformational, tear film abnormalities [4-6], Toxoplasma gondii [7] but it is most commonly associated with ocular injury and inflammation $[4,8]$ The condition is a distinctive corneal lesion observed particularly in felines [9]. The sequestrum typically presents itself as healing tissue in various shades of brown to black on the central or the paracentral cornea. Corneal irritation due to sequestrum may lead to blepharospasm, lacrimation and enophthalmos, protrusion of the third eyelid. Surgical removal by keratectomy is the preferred approach to relieve discomfort. Pharmacological management needs extended period for sequestrum to loosen and slough off. Causes and mechanisms leading to corneal sequestration remain undefined. Recurrence of the lesion can occur at the original site after surgical removal. Various studies have been conducted to identify the pathophysiology of feline sequestrum, based on the tissue analysis of the sequestrum. In this case report, we describe the success of surgical outcome; determine tissue changes in feline sequestrum and the recurrence at a new site following surgical resection.

\section{Materials and Methods}

A 4-year-old spayed female cat was examined for "a black spot" on the central cornea of left eye leading to ocular irritation and discomfort persistent for the past one month; owner also reported of excessive lacrimation for the initial few days. Owner revealed a history of corneal injury one month prior to the presentation while she was playing with other mates. The local veterinarian prescribed a topical antibiotic, and after one-month follow-up, the patient was referred to the centre. General examination was performed; pupillary reflex- direct and consensual was performed. Schirmer's tear test and fluorescein dye test was performed in both eyes. The affected eye was examined under slit lamp biomicroscopy.

Based on history and clinical findings the condition was diagnosed as corneal sequestrum and a surgery was planned to relieve the discomfort. Routine blood examination for blood count, serum biochemical evaluation for blood sugar, kidney function was performed prior to anaesthesia to ensure no abnormalities. Anaesthesia was induced with a combination of Xylazine HCL @ $1 \mathrm{mg} / \mathrm{kg}$ and Ketamine HCL @ $5 \mathrm{mg} / \mathrm{kg}$ IM, and Diazepam @ 0.2mg/kg IV. Protective eye gel was applied to the right eye, and the left eye was prepared for aseptic surgery. Topical tropicamide and proparacaine were applied for mydriasis and corneal anesthesia respectively. Under the magnification of operating microscope, the surgery was performed. A corneal forceps was used to hold the lesion that was noticed to be loose superiorly and attached to the broad base, an 11 No scalpel blade was used to perform lamellar keratectomy and dissect out the 
lesion from the base very cautiously. The sequestrum along with its base was removed, and a pigmented crater was left behind (Figure 1). A third eyelid bandage was done to protect the wound bed. The dissected sequestrum was preserved in $10 \%$ formalin solution for histopathological investigation.

The patient was prescribed topical drops i.e. ocular lubricants (Refresh tears) ${ }^{\circledR}$, antibiotic (Vigamox) $^{\circledR}$, mydriatics (Tropicamide) ${ }^{\circledR}$ and anti-inflammatory (Flur) ${ }^{\circledR}$, for one month. The third eyelid bandage suture was removed after one month when it was observed that the wound bed had healed well with mild scarring, but surprisingly there was another new similar lesion in the lower quadrant of the initial lesion.

\section{Results}

General examination revealed the cat was otherwise healthy apart from its ocular affection. The owner reported of intermittent nasolacrimal secretions at times. Pupillary reflex, direct and consensual was normal in both eyes. Schirmer's test values for the left and right eye were $12 \mathrm{~mm} / \mathrm{min}$ and $14 \mathrm{~mm} / \mathrm{min}$ respectively. Fluorescein dye test was negative in right eye and in the left eye faint staining was observed around the border of the lesion. Slit lamp biomicroscopy showed the lesion in the left

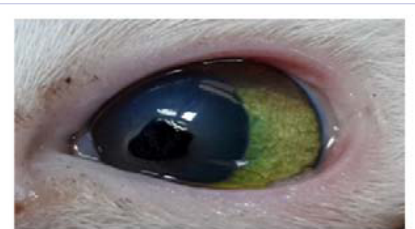

A

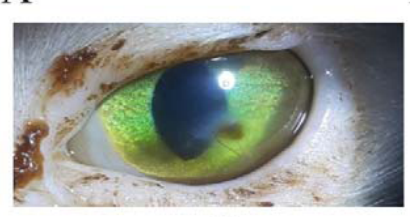

$\mathrm{C}$

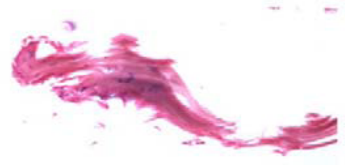

$\mathrm{D}$

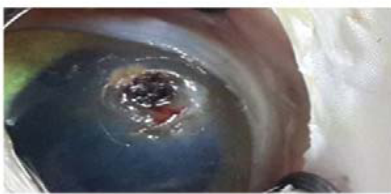

B

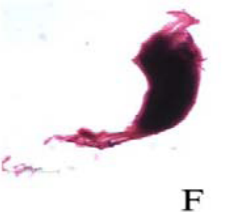

Figure 1: This figure shows the centrally positioned blackish corneal sequestrum (A), Surgical removal of the sequestrum leaves behind a pigmented crater like wound bed (B), Post operative appearance of the eye one month following surgery shows complete healing of the operated site with mild haze, however appearance of sequestra in the lower quadrant of the previous lesion is evident (C), Histopathological sections from the resected sequestrum shows thin epithelial layer with cells (D\&E), thick stromal fibrosis (F), no vascularisation or inflammatory cells are evident in these sections. eye had extended up to mid-stromal depth. Scanty and thin blood vessels were noticed around the lesion.

The surgery for removal of the sequestra was successfully performed. Histopathological sections of the sequestrum showed severe fibrosis of corneal stroma with a thin lining of epithelial cells. There was, however, no evidence of inflammation or vascularization within the resected tissue section.

The wound bed had healed well with mild scarring; however, recurrence of sequestrum had initiated in the lower quadrant of the primary site, which was an unusual finding.

\section{Discussion}

Corneal sequestrum is a remarkable and distinctive corneal affection reported in a feline, and its occurrence has been reported to have varied etiology. Amongst various other causative factors i.e. conformation, hereditary factor, feline herpesvirus -1 , toxoplasmosis and corneal injury has been identified as a major aetiology leading to corneal sequestration in feline [4]. The explanation by which each factor contributes to sequestrum formation is still unknown.

This study was an attempt to investigate further the surgical outcome as well as the histopathological features of the resected sequestrum. Considering the discomfort of the patient, a surgical resection was planned. The surgery was uneventful with no intra-operative complication. The wound bed appeared like a pigmented crater, most of the tissue had been removed. A third eyelid bandage was done to protect the wound bed; several techniques have been used to protect defect and enhance healing preventing recurrence i.e. corneal lamellar keratoplasty, conjunctival transposition, contact lens, etc. We tried this simple technique to reduce the time for surgery as the owner was not able to report for follow-up being located at a very distant place, we predicted it would not be possible to follow-up for conjunctival graft transposition.

Histopathological sections in our study showed stromal fibrosis and presence of a thin layer of epithelial cells; there was, however, no evidence of inflammation or vascularization of the tissue. In contrast to our findings previous histopathological studies on feline sequestrum report coagulative necrosis of corneal stroma, and heavy infiltration of inflammatory cells $[1,10]$. Studies involving the tissue characteristics of feline corneal sequestrum have reported the presence of iron [11], melanin [12] and mineralization of the tissue [13]. The varied etiological factors may incite the difference in tissue changes, therefore, need further investigation.

Recurrence of sequestrum over the previous site has been recorded, but there was no significant difference in the rate of recurrence with or without the use of a graft [12]. In our study, the previous surgical site had healed well with mild scarring but interestingly the recurrence of the lesion appeared on an adjacent site. There was no facility to evaluate the presence of feline herpes infection, in this case, which could have revealed the cause of recurrence in the different site. Feline herpes virus1 has a high clinical incidence, $90-97 \%$ cats are reported to be 
seropositive; moreover incidence of disease recrudescence is high $[14,15]$. Therefore, there is a possibility that the surgical procedure may have triggered an immune-mediated response to the viral antigen residing at the stroma leading to stromal keratitis and recurrence of a lesion in an adjacent area [15]. In the absence of a possibility of injury owing to the third eyelid bandage over the corneal surface, the above reasoning may be a possible explanation for fast recurrence during the study period. Further studies with larger number of cases are warranted to draw conclusions.

\section{Conclusion}

We hereby report the successful surgical management of feline corneal sequestration; stromal fibrosis was the only major pathological feature identified in the histological sections of the resected tissue. The most interesting observation was the recurrence of sequestrum at a different site while the previous surgical site healed with mild scarring.

\section{References}

1. Glaze MB, Gelatt NK. Feline ophthalmology. In: GELATT, N.K Veterinary ophthalmology. 3.ed. Philadelphia.: Lippincott Williams \& Wilkins; 1998. p.997-1052.

2. Stiles J, DcDermott M, Bigsby D, Willis M, Martin C, Roberts W, et al. Use of nested polymerase chain reaction to identify feline herpesvirus in ocular tissue from clinically normal cats and cats with corneal sequestra or conjunctivitis. Am J Vet Res 1997; 58(4):338-342.

3. Nasisse MP, Glover TL, Moore CP, Wigler BJ. Detection of feline herpesvirus 1 DNA in corneas of cats with eosinophilic keratitis or corneal sequestration. Am J Vet Ophthalmol. 1998; 59(7):856-858.

4. Morgan RV. Feline corneal sequestration: A retrospective study of 42 cases (1987-1991). J Am Anim Hosp Assoc 1994; 30:24-28.
5. Pentlarge VW. Corneal sequestration in cats. Compend Contin Educ Pract Vet. 1989; 11:24-32.

6. Andrew SE, Tou S, Brooks DE. Corneoconjunctival transposition for the treatment of feline corneal sequestra: A retrospective study of 17 cases (1990-1998). Vet Ophthalmol. 2001;4(2):107-11.

7. Cullen CL, Wadowska DW, Singh A, Melekhovets Y. Ultrastructural findings in feline corneal sequestra. Vet Ophthalmol. 2005;8(5):295303.

8. Zigler M. Feline herpetic keratitis. Eye Consulting Services. http:// www.eyevet.com/. 1999.

9. Arianne Pontes Oriá, Ana Maria Barros Soares, José Luiz Laus, Francisco de Assis Dórea Neto. Feline corneal sequestration. Cienc. Rural, 2001;31(3):553-56.

10. Pentlarge VW. Corneal sequestration in cats. In: Glaze MB. Ophthalmology in small animal practice. Trenton : Veterinary Learning Systems. 1996. p. 219-225.

11. Ejima H, Hara N, Kajigaya H. Detection of iron in a blackish lesion in a case of feline corneal sequestration. J Vet Med Sci. 1993;55(6):105152.

12. Featherstone HJ, Franklin VJ, Sansom J. Feline corneal sequestrum: laboratory analysis of ocular samples from 12 cats. Vet Ophthalmol. 2004;7(4):229-38.

13. Gemensky AJ, Wilkie DA. Mineralized corneal sequestrum in a cat. J Am Vet Med Assoc. 2001;219(11):1568-72.

14.David G. Feline herpesvirus-1: ocular manifestations, diagnosis and treatment options. Journal of Feline Medicine and Surgery. 2011;13:333-346. doi: 10.1016/j.jfms.2011.03.010.

15. Maggs DJ. Updates on Pathogenesis, Diagnosis and Treatment of Feline Herpesvirus Type 1. Clin Tech Small Anim Pract.2005;20(2):94-101. 